\title{
La dinámica del amor en el logos homilético de John Henry Newman
}

\author{
Dynamic of love in the homiletic
}

logos of John Henry Newman

Mauricio Albornoz

Artigo recebido em

28/09/2016

e aprovado em

07/11/2016.

V. $6-$ N. $12-2016$

* Doctor en teología dogmática por la Pontificia Universidad de Salamanca,

España. Profesor de Teología de la Universidad

Católica del Maule, Chile

\section{Resumen}

El pensador Inglés John Henry Newman va enfrentando las dificultades que suscitaba una determinada experiencia de fe religiosa. Tal cometido lo realiza a través de sus Sermones Parroquiales que domingo a domingo fue predicando en sus Iglesias de San Clemente; Santa María y Littlemore por un periodo de 19 años. A través de estos discursos logramos reconocer una serie de reflexiones que nos permiten proponer la idea de una naturaleza antropológica que propone la experiencia del amor como realidad necesaria para el logos creyente. Nuestro autor va describiendo progresivamente en el devenir del tiempo (1824-1843) una serie de formulaciones teológicas que irán posibilitando la elaboración de condicionantes que solicita el acto de fe, y describen la dinámica de este en el vínculo logos-amor. Lo que a su vez da cuenta de una determinada comprensión del 
ser humano. Esto es lo que proponemos. Creemos que nuestro autor en su predicación dominical va revelando una determinada comprensión del ser humano que pone en tensión la experiencia del amor con el logos propio de un discurso teológico, hasta situar esta tensión como característica fundante del acto creyente, lo que invita a la credibilidad de este mismo acto, y por lo mismo, presencia misteriosa del amante en el amado.

Palabras clave: Newman, amor, fe, antropología, sermón.

\section{Abstract}

The English thinker John Henry Newman is facing the difficulties raised a certain experience of religious faith. Such a task is performed through their Parochial Sermons Sunday to Sunday was preaching in their churches of Saint Clement's; Saint Mary's and Littlemore church for a period of 19 years. Through these speeches we recognize a series of reflections that allow us to propose the idea of an anthropological nature proposing the experience of love as necessary reality for the believer logos. Our author is describing progressively in the course of time (1824-1843) a series of theological formulations that will enabling the development of conditions requested by the act of faith, and describe the dynamics of this love-logos in realtionship. Which in turn realizes a certain understanding of human beings. This is what we propose. That is, we believe our author in his Sunday preaching is revealed a certain understanding of the human being that tenses the experience of love with their own logos theological discourse, to put this tension as a founding property of the believer act, inviting the credibility of this act, and therefore mysterious presence of the lover in the beloved.

Key words: Newman, Love, Faith, Antropology, Sermon.

\section{Introducción}

ntentar hablar de antropología o de lo que podemos comprender razonablemente del ser humano, siempre nos sitúa frente a una posibilidad inmensa que exigiría recorrer varios siglos de trabajo sistemático en el afán de intentar responder a la pregunta por el hombre. Y, haciéndolo, pudiese ser que no lo veamos con claridad; en ese sentido no resulta arbitrario que el Concilio Vaticano II se haya referido al hombre como misterio (mysterium; GS 22), o que la Exhortación apostólica post-sinodal Verbum Domini hable del enigma de la condición humana (hu- 
manae condicionis aenigma; VD 6). La complejidad que exije una comprensión de la realidad humana nos obliga a precisar nuestro estudio en una determinada comprensión del hombre que evidencie en sus propias facultades humanas -como condición de posibilidad-, la sobrenaturalidad de la fe, y las posibilidades del ser humano para alcanzar un asentimiento a ella que le permita vivir una verdadera experiencia religiosa.

La cuestión que proponemos no es baladí. Recordemos las palabras del entonces Cardenal Joseph Ratzinger al terminar su discurso en La Sorbona de Paris, el 27 de noviembre de 1999. Al hablar de la religio vera advertía: "su contenido deberá consistir, en lo más hondo, a decir verdad hoy como ayer, en que el amor y la razón coinciden como pilares fundamentales propiamente dichos de lo real: la razón verdadera es el amor y el amor es la razón verdadera. En su unidad, son el fundamento

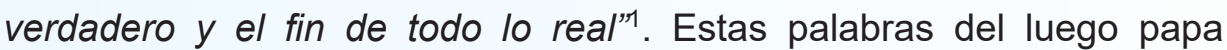
Benedicto $\mathrm{XVI}$, amor y razón, como fundamento de lo real en la religión, nos hablan de facultades humanas que sostienen la experiencia creyente, por lo que su lugar para la fe no es secundario, lo que hace necesaria la búsqueda de una elaboración más sistemática. En efecto, al hablar de estas facultades humanas nos adentramos en el problema antropológico, y si se tratan en razón de la fe religiosa, nos introducimos en la antropología teológica.

Dichas facultades en cuanto humanas nos remiten al hombre mismo, y todo lo que constituye al hombre es evidentemente antropología o al menos puede ser objeto de ella. Como cita Miguel de Unamuno ${ }^{2}$ recogiendo las palabras de Publio Terencio que en boca de Cremes decía en el año 165 antes de Cristo: "soy humano, por lo tanto, nada de lo humano me es ajeno" (Homo sum, humani nihil a me alienum puto) ${ }^{3}$,

1.J. Ratzinger, “¿Verdad del cristianismo?”, Revista de Historia, 2 (2000), 11-25, aquí, 25. 2.M. de Unamuno, Del sentimiento trágico de la vida. La agonía del cristianismo, Madrid $1983,57$.

3.Terencio, Comedias, el Heautontimorúmenos. Formión. I-77, vol. II, Salamanca 1991, 39. 
pues del mismo modo debe rezar la antropología teológica ${ }^{4}$.

En esta misma dirección hemos tomado nota de Tomás de Aquino, que al hablar de la naturaleza intelectual del hombre como imagen de Dios (Sth I, q.93, a.4), sitúa el ejercicio de esta naturaleza antropológica en las facultades de conocer y amar: dimensión de la razón y dimensión afectiva. En efecto, el Doctor angélico realiza varias acepciones en este lugar de la Summa teológica para hablar del hombre como imagen de Dios. Hace referencia a la condición actual del hombre que conoce y ama por la gracia, y da perspectiva a este hecho al proyectar la idea de plenitud de este conocimiento y de este amor del que se gozará en la gloria.

Esta misma clave antropológica la sigue el Concilio Vaticano II, que al hacerse la pregunta por el hombre, responde en clave bíblica con una formulación prácticamente idéntica a la que recogemos de Tomás: "La Biblia nos enseña que el hombre ha sido creado a imagen de Dios, con capacidad para conocer y amar a su Creador" (GS 12). Las citadas referencias hacia las facultades racionales y afectivas, resultan determinantes en el reconocimiento de la experiencia sobrenatural de Dios, y en concreto de la fe religiosa. El conocimiento, como elemento racionalmente requerido, nos pone en una clave espistemológica que requiere de una profundización (razón), y de igual modo la experiencia del amor humano en sus diversas manifestaciones sugieren una referencia a Dios (sentimiento). En su conjunto -amor en cuanto sentimiento, y conocimiento en cuanto razón- nos dan una clave antropológica que se transforma en camino ineludible hacia la realidad de Dios, más aún, al Dios revelado en Jesucristo.

Lo dicho hasta aquí resulta tan común en la teología de la fe que la Comisión teológica internacional, en el año 2004 recoge una reflexión sobre el hombre como imagen de Dios (imago Deo), en la cual, luego

4.Es la insistencia de Pedro Fernández en su apartado sobre la antropología teológica en: A. Cordovilla, La lógica de la fe. Manual de teología dogmática, Madrid 2013, 171274 , aquí 173. 
de hablar del dominio del hombre sobre el cosmos y su capacidad de existencia social, se refiere al "conocimiento de Dios y el amor a Dios" como elementos que encuentran su raíz en el imago Deo ${ }^{5}$. Más adelante, el mismo documento afirma que: "Las actividades que derivan de la comunión interpersonal del hombre y del servicio responsable, se refieren a las capacidades espirituales; intelectuales y afectivas" ( $\mathrm{n}^{\circ}$ 26). $Y$ en el $n^{\circ} 45$ del mismo documento, al hablar del daño producido por el pecado, señala que éste afecta la relación del hombre con los otros en cuanto divide sus dimensiones, dentro de las cuales, y las afirma en paralelo, se encuentran la razón y la emoción. En suma, lo que vemos aquí formulado -aún reconociendo que estamos en grados de certidumbre teológica distintas-, nos lleva en conjunto a hacernos la pregunta por estas facultades y su relación con la fe religiosa en una propuesta teológico-fundamental. Si la solicitud afectiva (amor) por una parte, y racional (logos) por otra, en cuanto constitutivas de la humana naturaleza deben reconocerse en el acto creyente, esto sugiere una mejor sistematización.

Con todo, la complejidad que exige una comprensión de la realidad humana nos obliga a precisar una propuesta ${ }^{6}$, en nuestro caso, la situamos en una determinada comprensión facultativa del ser humano: el logos. Esta posibilidad de comprensión solicita una necesaria vinculación a un amor fontal para abrirse -como condición de posibilidad-, a la experiencia sobrenatural de la fe, sin la cual la vida creyente carecería de consistencia, palideciendo en su rostro y eclipsando sus perspectivas. Esta es la propuesta que se devela confirmada en el discurso homilético de un autor anglicano en la primera mitad del siglo XIX, nos referimos por supuesto a John Henry Newman, nuestra figura.

El pensador Inglés va enfrentando las dificultades que suscitaba una

5.Cf. Comisión teológica internacional, "Comunión y servicio: la persona humana creada a imagen de Dios", del 23 de julio 2004, № 22. En BAC-documentos, Madrid 2009, 23.

6 . Es lo que sugiere Ladaria como clave metodológica al introducirse en estos temas de antropología. Cf.Ladaria, Luis. "Naturaleza y sobrenatural". en: Sesboüe, Bernard. Historia de los dogmas. El hombre y su salvación. 2010: 281. 
determinada experiencia de fe religiosa. Tal cometido lo realiza a través de sus Sermones Parroquiales que domingo a domingo fue predicando en sus Iglesias de San Clemente; Santa María, y Littlemore por un periodo de 19 años. A través de estos discursos, que suman un total de 604, logramos reconocer una serie de reflexiones que nos permiten aventurar la idea de una naturaleza antropológica que propone la experiencia del amor como realidad necesaria para el logos creyente.

\section{El camino hacia la fe}

Lo primero que hay que esclarecer, es que las ideas de Newman no buscaban resolver en un discurso formal las situaciones que le interrogaban, respecto de la comprensión creyente que le sobreviene, su perspectiva más bien intenta otorgar una cierta lógica pastoral que visibilice lo invisible de la fe religiosa. Por ello es que su discurso se entiende antropológico y práctico, buscando apelar al hombre entero, no sólo su racionalidad o su voluntad, por ejemplo, sino la totalidad de la experiencia humana. El paradigma que se abre en esta intencionalidad que deviene homilética, se circunscribe en dos ámbitos de comprensión; la idea de un logos y la dinámica del amor. Se trata, entonces, de que el sobrenatural de la fe se comprenda tan común a la humanidad que resulta imprescindible vincular el decir teológico con la comprensión antropológica como tal ${ }^{7}$. O, dicho en términos más simples, que la experiencia de la fe religiosa acontece o puede acontecer en la vida del ser humano corriente, simple y sencillo, con toda su fuerza y plenitud sobrenatural.

No se trata sólo de buscar argumentos y explicaciones exclusivamente racionales apelando a un logos particular de lo sobrenatural, ni despertar

7. "La cuestión del hombre y la respuesta que hayamos de darle no debe considerarse como un área de estudio distintas de las distantes áreas teológicas por lo que respecta al panorama que abarca y su problemática, sino que se identifican con el conjunto de la teología dogmática en cuanto tal". RAHNER, Karl. "Teología e Antropología”. vol. III. 1969: 45. 
sentimientos momentáneos que puedan eventualmente materializar un sentir y movilizar a un actuar. Lo que se buscaba es que sea el individuo en su totalidad el que entre en la acogida de una Palabra anunciada, y desde aquí experimenten su propia mismidad hacia lo trascendente dejándose transformar por ella, como destacaba Church al hablar de Newman, las "personas que escuchaban regularmente los sermones y sentían que eran diferentes a cualquier tipo de predicación, apenas reparaban en su influencia real o llegaban a advertir de momento el impacto que estaban ejerciendo sobre ellas" (1970, p. 92, 93).

El punto es que, siendo el individuo entero el que se compromete con la fe al asentir a ella, será la vida entera del mismo y su conocimiento total lo que se vuelque a Dios, y no sólo ideas, conceptos, sentimientos o emociones, por muy loables que estos sean ${ }^{8}$. Esta es la razón por la que nuestro autor buscaba llegar e involucrar las diversas facultades del ser humano, pues, estando la razón convencida y los sentimientos involucrados, el individuo necesariamente tiende a una determinada realidad que lo conduce e impulsa en un apriori de lo que se le presenta a seguir. El desafío era entonces de carácter antropológico vinculado a la fe religiosa, y este es quizá el motivo que matiza la problematización enfrentada en el discurso homilético de nuestro autor.

Esto es tan claro en el conjunto de la predicación de la figura newmaniana que si tuviésemos que definir cualitativamente las temáticas más trabajadas, considerando los sermones predicados mayor cantidad de veces, tendríamos que quedarnos básicamente con dos líneas de predicación, a saber, aquellas referente a los temas antropológicos y las que dicen relación con contenidos apologéticos respecto de la fe y su credibilidad. En términos más precisos digamos que sermones referentes a temáticas de antropología y que fueron predicados una pluralidad de veces tenemos los números: 53-83-124-190-232-257-258-

8. En este sentido no es accidental que, Vincent Ferrer Blehl, en la edición de su selección de Sermones de Newman, haya dado el título deRealizations, Newman's Own Selection of His Sermons. 
262-265-304-327-330-472 ${ }^{9}$. Y, entre aquellos referentes a discursos teológicos sobre fe y credibilidad, también predicados cuatro veces y más tenemos los números: 124-173-236-257-263-310-559. Esta cuestión ya sugiere la centralidad temática propuesta en nuestra comunicación y que mostramos en la gráfica siguiente (G.1).

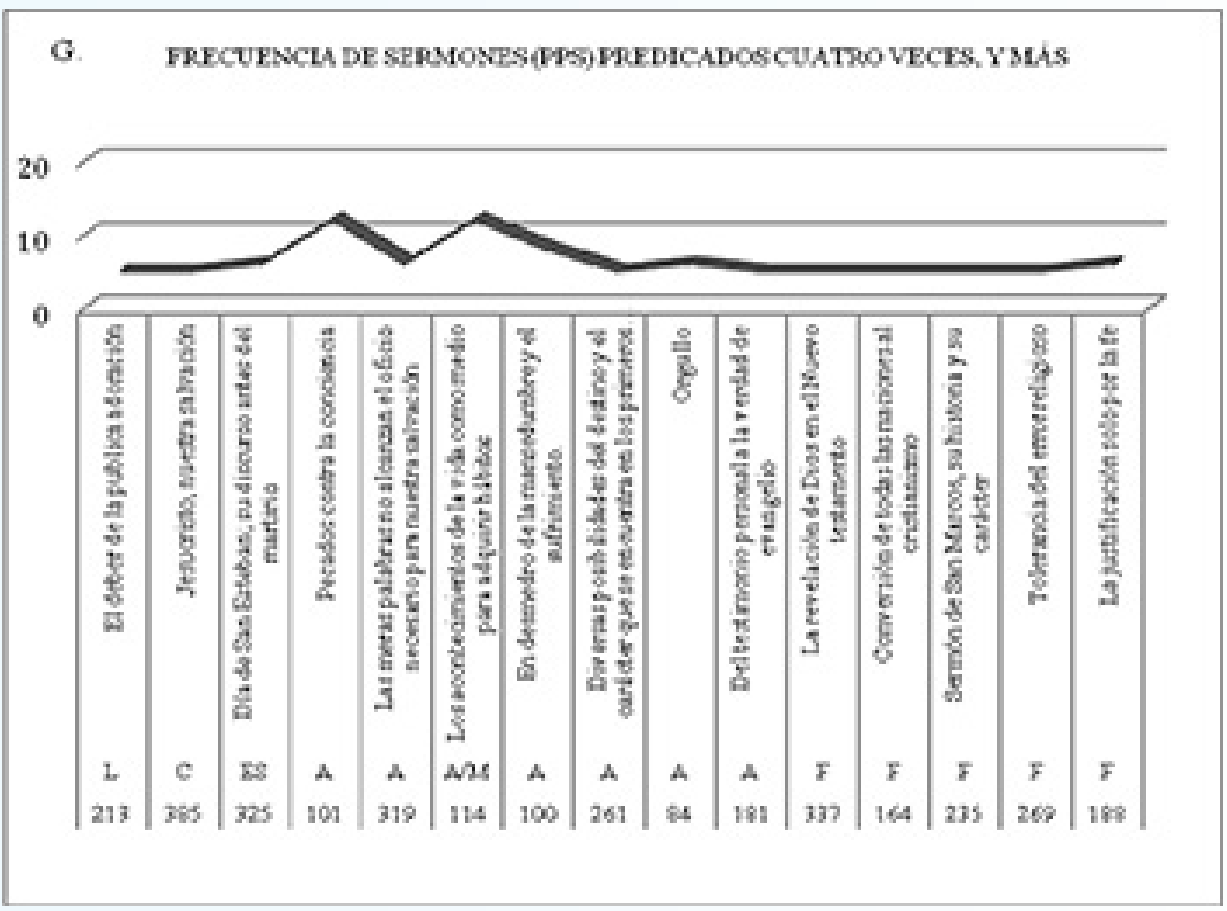

Hay que decir que los sermones no están pensados para convencer a aquel que no tiene fe, sino en llegar al creyente que con o sin conciencia debe enmendar sus caminos hacia una mayor "madurez cristiana", como

9.El número corresponde a una elaboración clasificada y codificada a nivel general de los sermones de Newman. Para confrontarlos se puede tomar como referencia, y con mayor complementación y detalle las listas de sermones de reciente publicación (ALBORNOZ, Luis. John Henry Newman y la condición antropológica como fundamento para la teología de la credibilidad,2015). 
se titula su sermón del 15 de mayo de $1831^{10}$. Por esta misma razón es que el predicador debe tener un punto definitivo ante él, con el que tiene que impactar a sus oyentes, y a partir del gráfico antecedente queda claro que estos puntos centrales giraron entorno a la idea de la fe y su fundamentación antropológica.

\section{Antropología y credibilidad}

Con los antecedentes citados podemos reconocer con cierta facilidad la natural percepción de los actos de fe en la vida ordinaria del hombre. Nuestro autor afirma que en el ámbito del conocimiento y el saber científico positivo, por ejemplo, los actos de fe se experimentan de modo semejante, es decir, tal como suceden en nuestra vida cotidiana. En efecto en la vida diaria confiamos permanentemente para proceder a la acción y la toma de decisiones y en el ámbito epistemológico la realidad acontece de igual manera. En consecuencia, para que el desarrollo científico avance es necesario confiar en que los postulados anteriores y que fundamentan un nuevo paso sean ciertos, de lo contrario cada paso en la ciencia supondría descubrir y experimentar nuevamente todos los postulados apriori y esto sería un absurdo. "La desconfianza, la falta de fe, rompe los lazos de la sociedad humana. Por eso, nos damos cuenta de que cuando uno no sabe de algo, lo único racional es fiarse de sus semejantes, o sea, ceder ante el juicio de otros por ser mejor que el propio"11. En otras palabras, "tenemos que confiar. Primero en los sentidos, luego en la memoria y en la razón; después, en otras autoridades. Así que, de hecho, casi todo lo que hacemos en la vida se

10.Cf. WHITE, W.D. "The Preaching of John Henry Newman”. 1969: 1-63... Introducción, parte primera, sobre la predicación en general. Complementando a esto pueden verse los números 135-175 de la Exhortación apostólica Evangelii Gaudium del Santo Padre Francisco que encontrará en su apartado sobre la homilía importantes coincidencias con el planteamiento homilético de nuestro autor.

11. "Distrust, want of faith, breaks the very bonds of human society. Now, then, shall we account it only rational for a man, when he is ignorant, to believe his fellow-man, nay, to yield to another's judgment as better than his own". NEWMAN, John Henry.Sermón: "La fe religiosa es racional". (n.196).P.S. p. 127. 
basa en la confianza; es decir, en la fe" ${ }^{m 12}$.

Sin embargo, tenemos que reconocer que todavía estamos aquí en el ámbito de la naturaleza, de la condición humana que hace posible la vivencia y convivencia de ideas y su desarrollo, diríamos que estamos aún en una base antropológica. La realidad descrita, en suma, es la que hace posible que acontezca otro estado de cosas, aquellas que pertenecen a lo sobrenatural, como es el caso de la fe religiosa ${ }^{13}$. Si bien esta última solicita de la primera, la trasciende, posibilitando, llevar más allá de lo estrictamente humano una cierta comprensión del ser humano en cuanto tal y del mundo que le rodea.

Estas reflexiones son las que nuestro autor destaca como experiencia religiosa auténtica o fe real, se trata de descubrir y vivir a partir de la fe religiosa la experiencia de la trascendencia que puede, eventualmente, llevar al ser humano a reconocer y vivir realidades incluso distintas a sus propias inclinaciones, cuando esto se da así es posible reconocer una fe real. Señala Newman: "La fe auténtica nos enseña a llevar a cabo un sinnúmero de cosas desagradables por amor a Cristo, a soportar las pequeñeces que nos fastidian, todo lo que no encontramos escrito en los libros. En la mayor parte de ellos la conducta cristiana se nos presenta grandiosa, elevada, espléndida. De modo que cualquiera que solamente derive su conocimiento de la religión verdadera de los libros, y no de los esfuerzos auténticos que implica ser religioso, seguramente se verá ofendido por la religión cuando se encuentre con ella de verdad, por la dureza y la humildad de sus obligaciones y las necesarias deficiencias

12. "Beyond this we must trust; and first our senses, memory, and reasoning powers; then other authorities: so that, in fact, almost all we do, every day of our lives, is on trust, i.e. faith". Ibidem. p. 125.

13. En este sentido el Concilio Vaticano II en la Constitución sobre la Divina Revelación; Dei Verbum, presenta la fe como un "encuentro personal con el Dios que se revela". Dei Verbum 5 . 
que existen al ponerla en práctica"14.

La distinción que Newman sitúa en el acontecimiento de la fe religiosa pone en evidencia la situación que intentamos presentar suscintamente, a saber, que la naturaleza antropológica implícitamente, requiere para poder alcanzar las realidades que la superan un acto de amor: pues la fe auténtica nos enseña a llevar a cabo un sinnúmero de cosas desagradables por amor a Cristo y es este sentimiento de amor lo que hace posible alcanzar realidades que la sola razón rechazaría.

En definitiva, se hace posible vivir las exigencias de una fe sobrenatural en la naturaleza (DV 5), porque hay un sentimiento que la mueve y que esa misma naturaleza reconoce. En este sentido se puede decir que la razón sigue al amor y ambas realidades en su conjunto posibilitan un asentimiento a la fe, como condición de posibilidad en su distinción epistemológica: "Dios ayuda al hombre a entender no solo por parte del objeto que lo propone para que lo entienda, o por aumento de luz, sino también porque procede de Dios la misma luz natural del hombre que le hace inteligente ${ }^{\Re 5}$.

La tensión existente entre ambos ámbitos de la fe, razón y amor, otorgan una base humana que permite (el hombre como capax Dei) en su condición de posibilidad, reconocer este acontecimiento sobrenatural (revelación), y abandonarse a él. Sin embargo, se debe admitir que la realidad sobrenatural de la fe religiosa lleva a juzgar permanentemente

14. "True faith teaches us to do numberless disagreeable things for Christ's sake, to bear petty annoyances, which we find written down in no book. In most books Christian conduct is made grand, elevated, and splendid; so that any one, who only knows of true religion from books, and not from actual endeavours to be religious, is sure to be offended at religion when he actually comes upon it, from the roughness and humbleness of his duties, and his necessary deficiencies in doing them. It is beautiful in a picture to wash the disciples' feet; but the sands of the real desert have no lustre in them to compensate for the servile nature of the occupation". NEWMAN, John Henry. Sermón: "El peligro de lograr las cosas". (n. 312). P.S.p. 464, 465.

15."Deus igitur ad intelligendum hominem iuvat non solum ex parte obiecti quod homini proponitur a Deo, aut per additionem luminis, sed etiam per hoc quod ipsum lumen naturale hominis quo intellectualis est a Deo est". DE AQUINO, Tomás. Opúsculos y cuestiones selectas. 2008:142. 
la realidad y la vida creyente bajo parámetros que se encuentran más allá de la naturaleza, es una tensión permanente.

¿Cómo es posible entonces acoger la fe religiosa si ésta camina por vías que superan la misma naturaleza humana? La condición antropológica lo hace posible, porque en el ser humano mismo se encuentran los elementos necesarios para que la fe sobrenatural pueda acontecer, los sentimientos humanos, específicamente el amor, puede reconocer una realidad que está más allá de sus propias posibilidades, y amándola, la razón le reconoce pudiendo asentir a ella, de lo contrario: 'Nos negamos a admitir una doctrina tan absolutamente distinta a las cosas que el mundo nos dice. Son hechos que no tienen paralelo alguno con nada conocido, pertenecen a un orden de cosas del todo nuevo y diferente, y mientras el corazón no tenga cercanía con ellos, la razón se niega a admitirlas"16.

Pero el lugar de la razón no se queda pasivo en la existencia del hombre. Ella -la razón-, está llamada a ser un instrumento eficaz en las distorsiones que la fe religiosa pueda enfrentar. $Y$ no solo puede dar instrumentos eficaces en la fundamentación de esta fe, sino también mostrarnos con claridad que la fe, para que se aprecie como religiosa, debe confiar en sus posibilidades, pues según lo señalado por Newman“También alguien puede decir: «estoy convencido de que tengo esa fe, siento que la tengo; siento que puedo apropiarme de los méritos de Cristo». O: «tengo la seguridad de que he sido perdonado». Es verdad; pero ¿dónde dice la Escritura que semejante seguridad, sin otro apoyo que nuestro sentirlo así, viene de Dios? ¿Dónde se promete eso? Hasta que no demos con ello, deberemos contentarnos con no

16."We refuse to admit a course of doctrine so utterly unlike any thing which the face of this world tells us of. These are events without parallels; they belong to a new and distinct order of things; and, while our heart has no sympathy with them, our reason utterly rejects them". NEWMAN, John. Sermón: "El Hijo encarnado sufrió y fue víctima expiatoria” . (n. 403). P.S. p.1237. 
estar seguros y, a la vez, temer y esperar acerca de nuestro destino"17.

\section{Conclusión}

El existencial de la fe se expresa en esta condición antropológica: sentimiento y racionalidad, o mejor "razón y amor", en donde encuentra su hogar, ahí está la recepción vital de su esencia, sin la cual, la propia condición humana se vería perturbada, desorientada y perdida ${ }^{18}$. No se puede entonces, diría nuestro autor, exigirle a la fe un axioma racional que por la propia naturaleza le trasciende: "Reflexionad un poco: ¿qué es la fe sino la aceptación de lo que no se ve, por amor, más allá de lo que determinan el cálculo y la experiencia? La fe supera al argumento"19. Para llegar a comprender lo que esta situación nos propone se solicita efectivamente de un análisis a la experiencia religiosa en la subjetividad humana, en donde se posibilita esta apertura trascendental a Dios en la propia inmanencia del hombre.

Esta es la novedad que fluye de la propuesta de nuestro autor, que sin manifestarlo explícitamente como un desarrollo antropológico —ni

17."But again, a person may say, "I have a conviction I have this faith; I feel I have; I feel I can appropriate the merits of Christ." Or again, "I have an assurance that I am forgiven." True; but where does Scripture tell us that such an assurance, without grounds for it beyond our feeling it, comes from God? where is it promised? till it is found there, we must be content not to be sure, and to fear and hope about ourselves at once". Ibidem. Sermón: "Paz y alegría en medio del castigo".(n. 415). P.S. p. 813.

18.Como una forma de mostrar las posibilidades en el uso de los conceptos que con nuestro autor hay que tener en cuenta, según hemos dicho, y por tanto la necesaria atención a los contextos en que se enuncian, valga esta aclaración que él mismo realiza: Hablando de la distinción entre la reverencia hacia el día del Señor y los deberes religiosos Newman señala que esto incumbe a la percepción moral cultivada (V; 2.2$)$, o "a lo que a veces impropiamente se denomina emoción (feeling). Impropiamente, porque la emoción viene y se va y, al no tener raíces en nuestra naturaleza, no se pronuncia con autoridad divina; en cambio la percepción moral, si bien varía en el conjunto de los seres humanos, es algo fijo en cada individuo, y es un elemento primordial dentro de nosotros" (Improperly, because feeling comes and goes, and, having no root in our nature, speaks with no divine authority; but the moral perception, though varying in the mass of men, is fixed in each individual, and is an original element within us). U.S., 60.

19."For only reflect, what is faith itself but an acceptance of things unseen, from the love of them, beyond the determinations of calculation and experience? Faith outstrips argument". NEWMAN, John Henry. Sermon. "La ley del espiritu". (n. 572). P.S. p.1347. 
anterior, ni posteriormente a los sermones-, se deduce de sus propias homilías. Pues la credibilidad del acto de fe supone una antropología, supone un hombre capaz de Dios: "No te atreves a pedir ahora su presencia; ¿la pedirías si vivieras los mismos años que Matusalén? Creo que no. Nunca serás suficientemente bueno como para desearla, nadie en toda la Iglesia la pide sin ciertas condiciones implícitas" 20 , y Newman nos va presentando esas condiciones implícitas progresivamente en esta condición antropológica que subyace en su discurso homilético.

Esta doble comprensión en razón de la condición antropológica que se nos presenta como antecedente a la credibilidad de la fe, manifiesta la tarea de que si bien el sentimiento y la razón posibilitan la acogida al acto de fe, estos a su vez también se solicitan en el desarrollo de la misma. Efectivamente, no son realidades que una vez que el acto de fe ha acontecido en el ser humano en particular desaparezcan, no son realidades transitorias sino que permanecen en el hombre cualquiera que sea su estado. La fe religiosa entonces encuentra en estas dimensiones sus posibilidades de reconocimiento y también de desarrollo. Esto resulta relevante para la práctica de la fe y la sistematización de la misma, para la vivencia de la devoción religiosa y para la teología. Si bien nuestro autor no lo plantea de este modo, de sus sermones se deduce esta comprensión antropológica en virtud del desarrollo de la propia fe: "Nos negamos obstinadamente a aceptarlo; sabemos que no somos irreligiosos del todo y eso nos convence de que somos religiosos. Desarrollamos la idea de que es posible ser demasiado religioso, nos hemos acostumbrado a pensar que la religión no tiene nada de elevado o profundo, que hay poco campo para el ejercicio de nuestras emociones, poco pasto para

20."You dare not pray for His presence now; would you pray for it had you lived Methuselah's years? I trow not. You will never be good enough to desire it; no one in the whole Church prays for it except on conditions implied". Ibidem. Sermón: "El miedo a la venida de Cristo". (n. 537). P.S. p. 992. 
nuestra inteligencia, poco espacio para nuestros esfuerzos" 21 .

Esta especie de conformismo que critica nuestro autor en una fe que prescinde de profundización religiosa en materia emocional y racional da cuenta de lo que señalábamos. Es decir, la fe no solo se construye en base a postulados previos que la sostienen sino que exige de ellos su propia profundización y desarrollo. Y en la medida en que este amor a la religión y el conocimiento de ella avance, avanzará también la fe: "Año tras año ganaremos algo, y cada pascua, cuando llegue, nos permitirá alegrarnos con más corazón y más conocimiento en la gran salvación que Cristo nos ganó"22. Esta convicción de nuestro autor en reconocer ambas realidades humanas que en la conciencia se transforman en constitutivas del acto de fe y el desarrollo de la misma van ponderando la simultaneidad que busca en la aplicación de las mismas realidades en el ejercicio creyente.

No se trata de que sea un aspecto el que se desarrolle, sino los dos, esto es lo que garantiza la experiencia de fe real. Si fortalecemos la dimensión racional en el creer y profundizamos en el conocimiento de la fe y su contenido, esto por sí mismo no nos entrega la salvación que se busca. Si no hay una experiencia de amor hacia Dios que nos lleve a la práctica de la fe y la mejor comprensión de ella, la realidad fundante en lo que antropológicamente podemos reconocer resulta debilitada en lo humano, y por tanto también en materia de fe religiosa: "Sin amor, podremos tener remordimiento, arrepentimiento; podremos reprocharnos cosas, pero eso no es penitencia, eso no nos salva. Puede

21."We know we are not altogether irreligious, and we persuade ourselves that we are religious. We learn to think it is possible to be too religious; we have taught ourselves that there is nothing high or deep in religion, no great exercise of our affections, no great food for our thoughts, no great work for our exertions". Ibidem. Sermón: "Vigilar" (Whatching). (n. 486). P. S. p. 945.

22. "Year by year we shall gain something, and each Easter, as it comes, will enable us more to rejoice with heart and understanding in that great salvation which Christ then accomplished". Ibidem. Sermón: "Es difícil darnos cuenta de los privilegios sagrados que recibimos". (n. 534). P.S. p. 1249. 
haber convicción de la razón, pero no conversión del corazón"23. La búsqueda del equilibrio de estas dimensiones humanas resulta ser el punto de inflexión a partir del cual la fe religiosa encuentra su status antropológico, y esto es lo que nuestra figura newmaniana quiere reconocer y mostrar a sus oyentes para que la razón no sobrepase las expectativas emocionales, ni la emoción anule el necesario ejercicio racional. De esta manera el contexto intelectual y religioso que Newman enfrentaba abre la comprensión a una fe religiosa capaz de sostenerse en una base antropológica fundante, que a su vez, encuentra su razón y sentido en el diálogo fontal recurrente que se produce en estas dimensiones, y si estos así tenemos la sobrenaturalidad introducida en la naturalidad de la humanidad; la presencia, misteriosa posible que evidencia el lugar presente del amante en el amado.

\section{BIBLIOGRAFÍA}

ALBORNOZ, Luis Mauricio. John Henry Newman y la condición antropológica como fundamento para la teología de la credibilidad. España: Ed. Univ. Salamanca,2015.

Concilio Vaticano II. Constitución sobre la Divina Revelación; Dei Verbum. Madrid: BAC., 2014.

CHURCH, Richard. The Oxford Movement, Twelve Years 1833-1845. London: Macmillan \& Co., 1970.

DE AQUINO, Tomás. Opúsculos y cuestiones selectas, vol. V. Madrid: BAC.,2008.

FERRER BLEHL, Vincent.Realizations, Newman's Own Selection of His Sermons. London: Longman Green \& Co., 2009.

LADARIA, Luis. "Naturaleza y sobrenatural", en: SESBOÜE, Bernard. (dir), Historia de los dogmas. El hombre y su salvación, vol.II, Salamanca: Secretariado Trinitario, 2010.

NEWMAN, John Henry. Sermón: "La fe religiosa es racional" del 24 de mayo de 1829. (n.196). P.S., 127. Madrid: Encuentro, 2007.

NEWMAN, John Henry. Sermón: "La fe religiosa es racional" del 24 de mayo de

23. "Without love there may be remorse, regret, self-reproach, self-condemnation, but there is not saving penitence. There may be conviction of the reason, but not conversion of the heart". Ibidem. Sermón: "El amor es lo único necesario". (n. 527). P. S. p. 1170. 
1829. (n.196). P.S., 125. Madrid: Encuentro, 2007.

NEWMAN, John Henry. Sermón: "El peligro de lograr las cosas" (The Danger of Accomplishments) del 18 de octubre de 1831. (n.312). P.S., 464-465. Madrid: Encuentro, 2007.

NEWMAN, John Henry. Sermón: "El Hijo encarnado sufrió y fue víctima expiatoria" (The Incarnate Son A Sufferer and Sacrifice) del 24 de abril de 1836. (405).P.S., 1237. Madrid: Encuentro, 2013.

NEWMAN, John Henry. Sermón: “Paz y alegría en medio del castigo" (Peace and Joy Amid Chastisement) del 12 de junio de 1836. (n. 415). P.S., 813. Madrid: Encuentro, 2010.

NEWMAN, John Henry. Sermón: "El miedo a la venida de Cristo" del 4 de diciembre de 1836. (n. 437). P.S., 992. Madrid: Encuentro,2011.

NEWMAN, John Henry. Sermón: "Vigilar" (Whatching) del 3 de diciembre de 1837. (n. 486). P.S., 945. Madrid: Encuentro, 2010.

NEWMAN, John Henry. Sermón: "Es difícil darnos cuenta de los privilegios sagrados que recibimos" del 31 de marzo de 1839. (n. 534). P.S., 1249. Madrid: Encuentro, 2013.

NEWMAN, John Henry. Sermón: "El amor es lo único necesario" del 10 de febrero de 1839.(n. 527). P.S., 1170. Madrid: Encuentro, 2011.

RAHNER, Karl. "Teología e Antropología". vol. III, Roma. Nuovi Saggi. 1969.

WHITE, W.D. "The Preaching of John Henry Newman". Philadelphia. Fortress Press,1969. 